\title{
Rosuvastatin for enhancement of aneurysm neck endothelialization after coil embolization: promotion of endothelial progenitor cells in a rodent model
}

\author{
Peixi Liu, MD, ${ }^{1}$ Qingzhu An, MD, PhD, ${ }^{1}$ Xi Chen, MD, ${ }^{1}$ Jun Huang, MD, ${ }^{3}$ Guo-Yuan Yang, MD, PhD, ${ }^{2,3}$ \\ and Wei Zhu, MD, PhD'1 \\ 1'Department of Neurosurgery, Huashan Hospital of Fudan University; ${ }^{2}$ Department of Neurology, Ruijin Hospital, Shanghai Jiao \\ Tong University School of Medicine; and ${ }^{3}$ Neuroscience and Neuroengineering Research Center, Med-X Research Institute and \\ School of Biomedical Engineering, Shanghai Jiao Tong University, Shanghai, China
}

\begin{abstract}
OBJECTIVE Coil embolization is a safe, efficient, and minimally invasive technique for the treatment of intracranial aneurysms. However, coil embolization is associated with a higher risk of recurrence than clip ligation. In this study, the authors explore a new approach through the promotion of endothelial progenitor cells (EPCs) to optimize endothelialization of the aneurysm neck and reduce the risk of recurrence.

METHODS A coiled aneurysm model was created in 48 adult male Sprague-Dawley rats via microsurgery. Half of these animals were treated with rosuvastatin $(20 \mathrm{mg} / \mathrm{kg}$ ) in saline via gavage for 10,20 , or 30 days. The other half were administered saline without rosuvastatin. An additional 15 rats underwent "mock surgery" (identical anesthesia and saline gavage but no surgery). The endothelial repair process in the coiled aneurysms was evaluated via flow cytometry, immunostaining, and electronic microscopy. The mock surgery group was used for comparison in flow cytometry studies. The effects of rosuvastatin on viability and functioning of Sprague-Dawley rat bone marrow-derived EPCs were also explored via MTT, migration, and tube formation assays.

RESULTS The aneurysm neck repair score was significantly higher in the rosuvastatin-treated rats than in the untreated rats $(p<0.05)$. The circulating EPC count was increased and maintained at a higher level in rosuvastatin-treated rats compared with the aneurysm rats that did not receive rosuvastatin $(p<0.05)$. Immunostaining showed that the aneurysm neck endothelium was more integrated and the number of kinase insert domain receptor-positive cells was increased in the rosuvastatin-treated rats. Further study demonstrated that rosuvastatin promoted EPC proliferation, migration, and tube formation.
\end{abstract}

CONCLUSIONS Rosuvastatin promoted endothelialization of the coiled aneurysm neck via induction of EPCs, suggesting that promoting endothelialization provides an additional therapeutic opportunity during vascular endothelium repair.

http://thejns.org/doi/abs/10.3171/2015.3.JNS142841

KEY WORDS aneurysm; endothelial progenitor cells; endothelialization; rosuvastatin; vascular disorders

$\mathrm{I}$ $\mathrm{N}$ the treatment of intracranial aneurysms, coil embolization is safer, quicker, more efficient, and less invasive than clip ligation. Incomplete endothelialization of the aneurysm neck after coil placement, however, is associated with an increased risk of aneurysm recurrence and even rupture. Improving endothelialization of the aneurysm neck may therefore improve the long-term outcome for patients treated with coil embolization.
Endothelial progenitor cells (EPCs) were initially identified from human peripheral blood and were found to express CD34 and kinase insert domain receptor (KDR). ${ }^{2}$ Sources of EPCs include adult bone marrow, peripheral blood, umbilical cord blood, and tissue resident cells. ${ }^{4}$ EPCs can exist for a relatively long period within the circulating blood, serving to maintain vascular homeostasis and being involved in vascular repair. Circulating EPCs

ABBREVIATIONS DMSO = dimethylsulfoxide; $\mathrm{EPC}=$ endothelial progenitor cell; $\mathrm{GDC}=$ Guglielmi detachable coil; $\mathrm{H} \& \mathrm{E}=$ hematoxylin and eosin; $\mathrm{KDR}=$ kinase inert domain receptor; MTT = 3-(4,5-dimethylthiazolyl-2)-2,5-diphenyltetrazolium bromide; OD = optical density; SEM = scanning electron microscope; vWF = von Willebrand factor.

SUBMITTED January 3, 2015. ACCEPTED March 27, 2015.

INCLUDE WHEN CITING Published online September 25, 2015; DOI: 10.3171/2015.3.JNS142841. 
are an interesting area for research because of their clinical application potential, and they are an independent risk marker for future cardiovascular events. ${ }^{8}$ Studies involving animal models and human clinical studies have indicated that EPC transplantation can improve the functional recovery of the heart and limbs after ischemic injury. ${ }^{22}$

Experimental studies of aneurysms have shown that the endovascular injection of EPCs can improve aneurysm neck neointima formation in a rabbit aneurysm model. ${ }^{1}$ In addition, we have previously shown that bone marrow-derived EPCs play a crucial role in the closure and reconstruction of the aneurysm neck after coil embolization. ${ }^{30}$ EPCs have demonstrated a robust effect on aneurysm neck endothelialization and the prevention of recurrence. Because EPCs are involved in the vascular repair and remodeling process, EPC-mediated therapy has been proposed as a potential treatment for vascular disease. Clinical studies have also shown that specific vascular diseases are correlated with EPC deficiencies and dysfunctions. ${ }^{11,18}$ Increasing the local level of EPCs may therefore provide a new opportunity for aneurysm neck endothelialization.

Statins are a class of drugs that are used to lower cholesterol levels by inhibiting the enzyme HMG-CoA (3-hydroxy-3-methylglutaryl-coenzyme A) reductase. Rosuvastatin is a statin family member that is used to treat high cholesterol levels and related conditions and prevent cardiovascular disease. Previous studies have shown that statins affect several processes involved in vascular repair and remodeling, including angiogenic and angiostatic responses. ${ }^{9}$ It is largely unknown, however, whether statins stimulate angiogenesis and vasculogenesis through the promotion of EPC function.

Because of the importance of endothelialization in aneurysm treatment, we explored the effect of rosuvastatin on EPC mobilization and activity in the treatment of intracranial aneurysms.

\section{Methods}

\section{Experimental Protocol and Rat Coiled Aneurysm Model}

The animal protocol used in this study was approved by the institutional animal care and use committee of Fudan University, and the experimental protocol was reviewed and approved by the university's ethics committee. Sixty-three adult male Sprague-Dawley rats (Shanghai Slac Laboratory Animal Co. Ltd.) weighing 200 to 250 $\mathrm{g}$ were randomly divided into 3 groups for the in vivo portion of this study as follows. 1) Animals in the "aneurysm rat treated with rosuvastatin" group (AN-Rosu group, $\mathrm{n}=24$ ) were anesthetized with isoflurane using a vaporizer (Matrx, Midmark). The model was established using surgical microscopy (Leica Microsystems), and vasotransplantation was performed on the rats to induce aortic artery aneurysms as previously described. ${ }^{10,23} \mathrm{~A}$ vessel segment was obtained from a healthy rat abdominal aorta and used as a donor segment. The anastomosed aneurysm developed in the abdominal aorta, between the renal vein and iliolumbar vein. The proximal area of the aneurysm was constricted by partial ligation to create an aneurysm neck, and the distal region of the anastomosing vessel segment was completely ligated. A coiled aneurysm model was created via Guglielmi detachable coil (GDC) embolization prior to distal ligation. The AN-Rosu group was treated with $20 \mathrm{mg} / \mathrm{kg}$ rosuvastatin suspension saline liquid via gavage each day for 10,20 , or 30 days. 2) Animals in the "aneurysm rat treated without rosuvastatin" group (AN group, $\mathrm{n}=24$ ) also underwent microsurgery as above; the aneurysms were coiled as in the AN-Rosu group, and the animals were treated with an equal amount of saline by gavage each day, but they did not receive any rosuvastatin. 3) Animals in the mock-surgery group (MS group, $\mathrm{n}=15$ ) received inhalation anesthesia with isoflurane like the aneurysm groups but were not subjected to any actual surgery. These animals were subsequently administered saline via gavage each day (as in the AN group) (Fig. 1).

A 3-T MRI system (Magnetom Verio, Siemens Healthcare) was used for 3D MR angiography to detect aneurysm formation and coiled condition at 3 days after model establishment in the AN-Rosu and AN groups.

\section{Pathological Assessment}

Thirty days after the anastomosis procedure, the coilembolized aneurysms obtained from 6 rats in each group were resected under inhalation anesthesia and fixed in $4 \%$ formalin solution. After resection of the aneurysm and while still anesthetized, the rats were killed by means of cervical dislocation. After the sample was embedded in paraffin, a low-speed saw (SYJ-150 Kejing Automation Equipment Co.) was used to section the distal part of the coiled aneurysm as previously described. ${ }^{5}$ Coil fragments were removed carefully under microscopy, and the sections were re-embedded in paraffin. Then, the vascular tissue was sectioned again into 5- $\mu \mathrm{m}$-thick slices using a microtome (Model RM2165, Leica Microsystems). The sections were floated in a water bath $\left(42^{\circ} \mathrm{C}\right)$, mounted on Superfrost Plus slides (Fisher Scientific International), and dried overnight in an oven at $37^{\circ} \mathrm{C}$ (Fig. 2A). Three sections were randomly chosen for hematoxylin and eosin $(\mathrm{H} \& \mathrm{E})$ staining. For immunohistochemical analysis, anti-KDR (Abcam), antivon Willebrand factor (vWF) (Abcam), and anti- $\alpha$-smooth muscle actin ( $\alpha$ SMA) (Abcam) were used as primary antibodies and 3,3'-diaminobenzidine (DAB) plus chromogen (Thermo Fisher Scientific) was used for substrate visualization, according to the manufacturers' protocol.

After $\mathrm{H} \& \mathrm{E}$ staining, the morphological changes in the neck of the experimental aneurysm (proximal to the parent artery) were assessed. Based on the morphological characteristics, we established an aneurysm repair score consisting of aneurysm neck integrity and luminal endothelial layer continuity. For the integrity assessment, a value of 3 indicated a complete occlusion and a flat aneurysm neck, a value of 2 indicated a complete occlusion and small "dog ears," a value of 1 indicated a complete occlusion and large "dog ears," and a value of 0 indicated recanalization. With respect to endothelial layer continuity, a value of 3 indicated complete endothelium continuity, a value of 2 indicated obvious but small defects, a value of 1 indicated large defects, and a value of 0 indicated no endothelium formation (Table 1, Fig. 2B). We randomly chose 3 sections from each sample and the score of each sample was the mean of the 3. During assessment, the researcher estimator was blinded. 

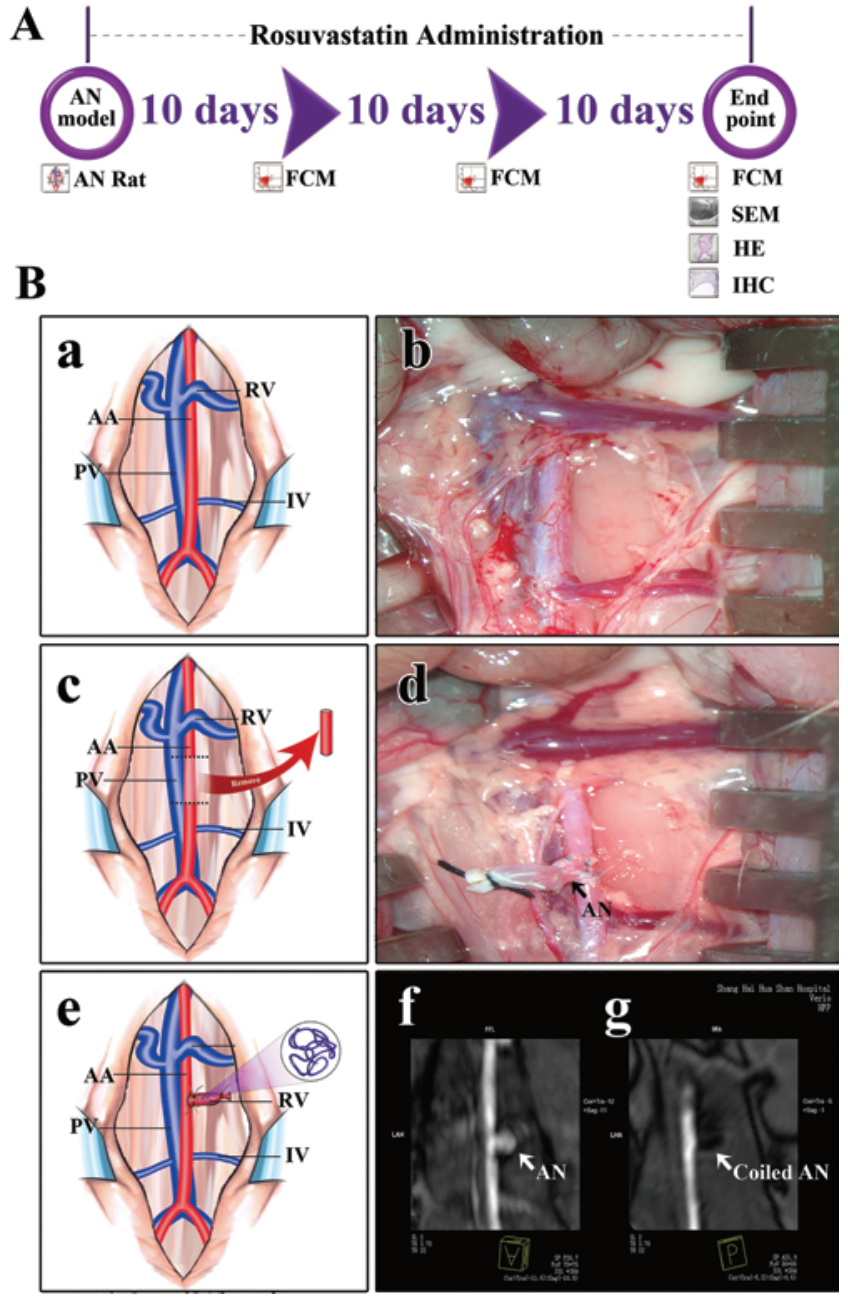

FIG. 1. Experiment design and the establishment of an aneurysm (AN) model. A: Schematic of experiment design. Rats with an experimental aneurysm treated with coil therapy and end-point examination via flow cytometry (FCM), scanning electron microscopy (SEM), H \& E staining $(\mathrm{HE})$, and immunohistochemical analysis (IHC) after 10,20, and 30 days of rosuvastatin treatment (AN-Rosu group) or saline administration (AN group). B: Photographs showing aneurysm establishment. The aneurysm was located between the renal vein (RV) and iliolumbar vein (IV) ( $a$ and $b$ ). The donor segment was connected to the abdominal aorta (AA) (c-e). The success of the rat aneurysm model was identified using MRI (f). Coils were placed after 3 days of aneurysm formation (g). PV = postcaval vein.

\section{Scanning Electron Microscope}

A scanning electron microscope (SEM, Hitachi SU8000) was used to evaluate the endothelialization of the aneurysm neck. After 30 days of gavage, the aneurysm area on the abdominal artery was removed from 3 animals in each of the 2 aneurysm groups, and the exposed luminal surface of the aneurysm neck was cut off from the parent artery along a longitudinal line. (The procedure was performed under inhalation anesthesia, and the animals were killed by cervical dislocation afterward.) The 6 vessel segments were fixed in $2.5 \%$ glutaraldehyde and processed for en face SEM imaging. The morphological membrane coverage of the orifice of the aneurysm neck and the endothelial cells adhering to the aneurysm neck were detected using a cold field emission SEM. The endothelial cells were identified as sheets of closely connected monolayer cells with a spindle or polygonal shape.

\section{Flow Cytometry}

CD34+/KDR+ cells were defined as EPCs. At Day 10, 20 , or 30 after aneurysm formation, the circulating blood was drained from 5 rats in each group via the carotid artery. (The rats were subsequently killed by cervical dislocation under anesthesia.) The rat blood was immediately stored at $4^{\circ} \mathrm{C}$. The mononuclear cells were then isolated via Ficoll (Histopaque-1077, Sigma) density gradient centrifugation within 4 hours after blood collection. The CD34 and KDR antibodies were added into the mononuclear suspension and incubated for 1 hour. After washing, the cells were labeled using Alexa Fluor 546 donkey anti-sheep IgG (Invitrogen) and Alexa Fluor 488 donkey anti-rabbit IgG (Invitrogen). After 30 minutes of incubation, the cells were washed and then analyzed using a flow cytometer (FACSAria II, Becton Dickinson) by collecting 300,000 events. The data were analyzed using FACSDiva Software. Gates were set on the forward- and side-scatter (FSC/SSC) plot corresponding to select mononuclear cells followed by the CD34+/KDR+ gate. The positive cells were compared with the negative isotypes from the control rats, and the results were expressed as a percentage of the mononuclear cell events.

\section{EPC Isolation, Culture, and Identification}

An additional 30 adult male Sprague-Dawley rats weighing 200 to $250 \mathrm{~g}$ were used for the in vitro portion of this study. Under inhalation anesthesia, the animals' femurs were removed, and the animals were then killed by cervical dislocation. Bone marrow cells were isolated from rat femur marrow via Ficoll density gradient centrifugation and then cultured on fibronectin-coated dishes with EGM-2 (Lonza) medium at $37^{\circ} \mathrm{C}$ in a $5 \% \mathrm{CO}_{2}$ incubator. The attached cells were used for DiI-AcLDL (Invitrogen) and FITC-UEA-I (Sigma) staining. The adherent primary cells were incubated with $15 \mu \mathrm{g} / \mathrm{mL}$ DiI-AcLDL for 4 hours at $37^{\circ} \mathrm{C}$. The cells were fixed with $10 \%$ paraformaldehyde for 10 minutes and counterstained with FITC-UEA-I. For flow cytometry analysis, adherent primary cells (10th day) were resuspended, and KDR and CD34 antibodies were added to the mononuclear suspension, which was then incubated for 1 hour. After the cells were washed, they were labeled with Alexa Fluor 546 donkey anti-sheep IgG and Alexa Fluor 488 donkey anti-rabbit IgG. The cells were washed and analyzed using a FACSAria II flow cytometer by collecting 10,000 events. The data were analyzed using FACSDiva software.

\section{Cell Proliferation, Migration, and Tube Formation Assay}

Rosuvastatin calcium was dissolved in dimethylsulfoxide (DMSO, Sinopharm Chemical Reagent Co.) and filter sterilized. The rosuvastatin solution was diluted in the EGM-2 culture medium to different concentrations based on the experimental requirements.

EPCs were transferred to 96 -well plates at a cell density 

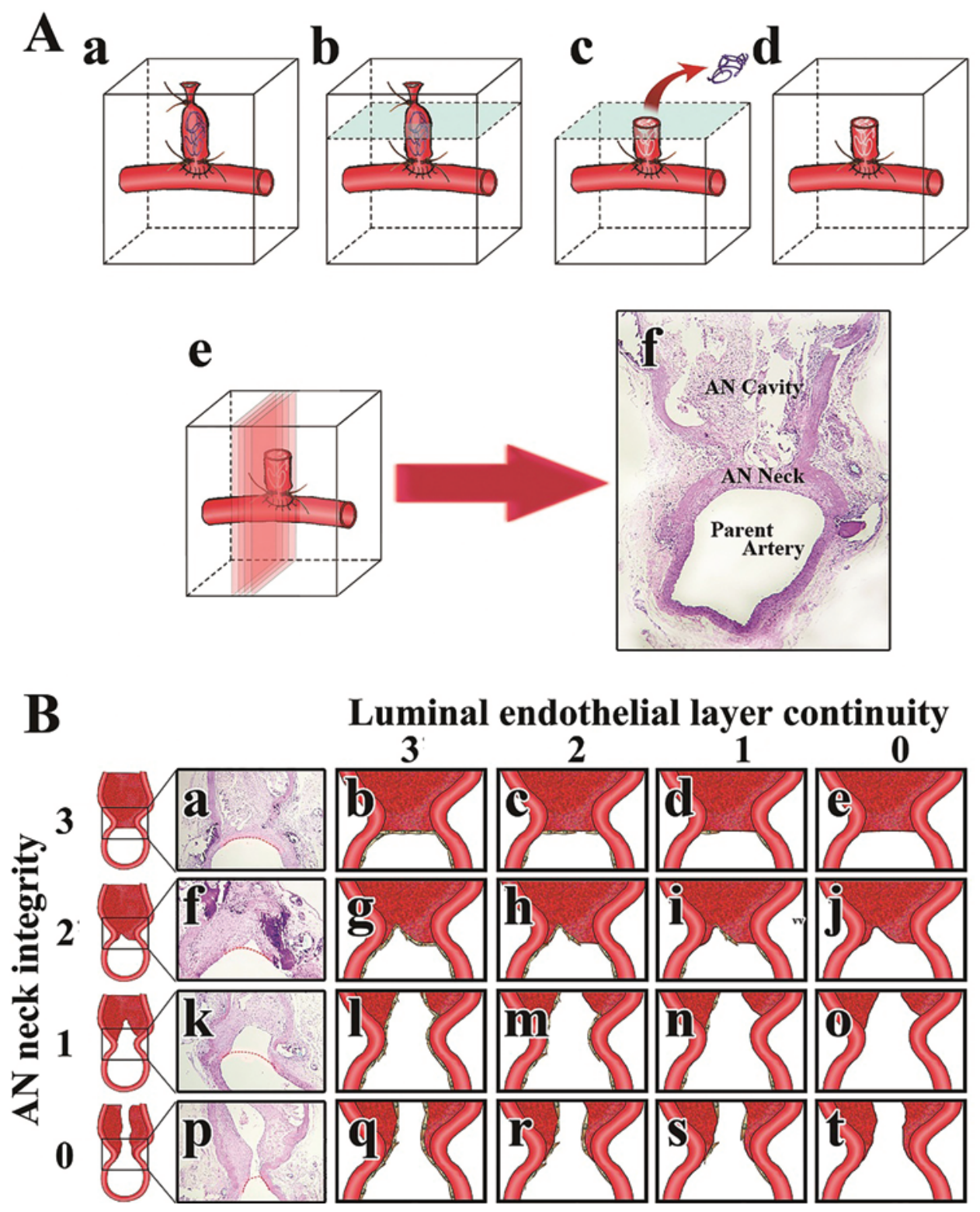

FIG. 2. Coiled aneurysm progress and aneurysm repair score assessment. A: Schematic illustration showing the coiled aneurysm tissue with parent artery in a paraffin block and the initial sectioning (a and b). The coil fragments were removed (c), and the tissue was re-embedded (d) and then cut into sections (5- $\mu \mathrm{m}$ thick) perpendicular to the long axis of the parent vessel. B: Photomicrographs of $\mathrm{H} \& \mathrm{E}$-stained sections $(\mathbf{a}, \mathbf{f}, \mathbf{k}$, and $\mathbf{p}$ ) and schematic illustration of aneurysm repair scoring system. Each row indicates a score from 0 to 3 for aneurysm neck integrity, and each column represents scores from 0 to 3 for luminal endothelial layer continuity. The total score was obtained from the sum of the 2 scores. The red dotted lines on the photomicrographs mark the aneurysm neck.

of $5 \times 10^{4}$ cells per well. After 24 hours, the cell culture medium was replaced with culture medium containing rosuvastatin at one of the following concentrations: 0.001 , $0.01,0.1,1,10$, and $100 \mu \mathrm{mol} / \mathrm{L}$. The cells were incubated for another 24 hours. Then, the culture medium in each well was removed and replaced with $10 \mu \mathrm{l}$ yellow tetrazolium MTT (3-[4,5-dimethylthiazolyl-2]-2,5-diphenyltetrazolium bromide) and $90 \mu \mathrm{l} \mathrm{EGM-2}$ medium. After 4 hours of incubation, the MTT culture medium in each well was removed and replaced with $100 \mu \mathrm{L}$ DMSO. The plates were placed in a rocking incubator for 10 minutes. Finally, the 96-well plates were analyzed using a microplate reader (BioTek) at $490 \mathrm{~nm}$. Changes in the optical density (OD) were used to evaluate the viability of EPCs treated with different concentrations of rosuvastatin.

For the migration assay, the EPCs were placed into 24well plates and incubated for 48 hours. Then, a pipette tip was used to scratch the cell monolayer across the center of each well. The detached cells were removed via washing, and the remaining adherent EPCs were treated with rosuvastatin $(100 \mu \mathrm{mol} / \mathrm{L})$ for 24 hours. The cells were then fixed, and propidium iodide (BD Pharmingen) stain- 
TABLE 1. Endothelium closure assessment system

\begin{tabular}{cc}
\hline \multicolumn{1}{c}{ Item } & Score \\
\hline Aneurysm neck integrity & \\
\hline Complete occlusion \& flat aneuysm neck & 3 \\
\hline Complete occlusion \& small "dog ears" & 2 \\
\hline Complete occlusion \& large "dog ears" & 1 \\
\hline Recanalization & 0 \\
\hline Luminal endothelial layer continuity & \\
\hline Complete endothelium continuity & 3 \\
\hline Obvious but small defect & 2 \\
\hline Large defect & 1 \\
\hline No endothelium formation & 0 \\
\hline
\end{tabular}

ing was performed. Fluorescence images were acquired, and the fluorescent area (\%) of the scratched region was determined.

For the tube formation test, EPCs were pretreated with $100 \mu \mathrm{mol} / \mathrm{L}$ rosuvastatin for 24 hours. The individual wells of a 96-well plate were coated with $30 \mu$ l Matrigel basement membrane matrix (BD Biosciences). Next, $3 \times$ $10^{5}$ rosuvastatin-treated EPCs were gently added to each gel-coated well. After 20 hours, the cells were dyed with calcein AM and examined via fluorescence microscopy. Images were acquired and analyzed for tube formation using the Wimasis image analysis software program. The total number of nets (distinct regions of tubes that contain at least 1 branching point) was used to estimate the level of tube formation. Isolated tubes were not considered as nets.

\section{Statistical Analysis}

The statistical analysis was performed using IBM SPSS Statistics version 19, and graphs were generated in GraphPad Prism version 5.01. Two-way ANOVA tests were used to analyze the percent of circulating EPCs (monocytes) identified by flow cytometry. One-way ANOVA tests were used to analyze the change in the OD value (\%) in the MTT assay, the fluorescent area (\%) in the migration assay, and the total number of nets in the tube formation assay; $p$ values less than 0.05 were considered statistically significant.

\section{Results}

\section{Rat Aneurysm Model}

After surgical vascular anastomosis, a rat aneurysm model was created. The surgery was performed in a total of 50 rats, but 2 rats died as a result of anesthetic overdose (the mortality rate was 4\%). The aneurysm was filled with circulating blood after surgery, and MR angiography confirmed the success of aneurysm formation in all 48 surviving animals. Finally, MR angiography performed 3 days later confirmed that the aneurysm was completely coiled (Fig. 1).

\section{Histological Findings}

$\mathrm{H} \& \mathrm{E}$ staining demonstrated that a more integrated aneurysm neck was formed and more spindle-like slender cells were present in the aneurysm necks of the AN-Rosu rats. In the AN rats, the intima of most of the aneurysm necks showed different degrees of a dog ear shape, and many small areas of cellular hyperplasia were also detected proximal to the aneurysm. The aneurysm repair score was significantly higher in the AN-Rosu group than in the AN group $(5.7 \pm 0.14$ vs $4.4 \pm 0.40, p<0.05$, Fig. 3A). Many KDR+ cells were detected in the inner surface of the aneurysm neck in the AN-Rosu rats; however, few $\mathrm{KDR}+$ cells were detected in the rats of the AN group. Further study demonstrated that there was a vWF+ cell layer in the aneurysm neck in both the AN-Rosu group rats and the $\mathrm{AN}$ group rats, suggesting that an endothelial cell layer formed in both groups. However, the vWF layer in the AN-group animals exhibited a lack of continuity (Fig. 3B and C). We also examined the lumen of the aneurysm sac. We found that KDR, $v W F$, and $\alpha$ SMA expression were increased in the rosuvastatin-treated (AN-Rosu) rats compared with the AN group rats, suggesting that growth in the lumen occurred (Figs. 3D-F).

\section{Scanning Electron Microscopy Examination}

Scanning electron microscopy showed the level of endothelialization in the aneurysm neck, and we found better endothelium coverage in the rats in the AN-Rosu group than in the rats in the AN group; this improvement primarily consisted of simple squamous epithelial cells at the bottom of the aneurysm neck. Overall, the rats that did not receive rosuvastatin demonstrated a similar sealing effect compared with the rosuvastatin-treated rats. However, some of the aneurysms were closed primarily with fibroblasts and smooth muscle cells at the bottom of the aneurysm necks. Endothelial cells were rarely observed in the AN group, and when present, they displayed long, flat, and fusiform morphological characteristics (Fig. 3D).

\section{Flow Cytometry}

On the 10th day after aneurysm formation, the number of circulating EPCs was significantly elevated. The number of circulating EPCs in both groups of rats with aneurysms (both treated and untreated) was different from that in the MS group by 10 days of treatment. The circulating EPCs showed no significant difference on the 20th day. On the 30th day, the level of circulating EPCs was mainly elevated by rosuvastatin treatment instead of aneurysm formation, and AN-Rosu rats showed a significant increase in the number of circulating EPCs after 30 days of treatment compared with the other 2 groups $(\mathrm{p}<0.05$, Table 2, Fig. 3E).

\section{EPC Isolation and Identification}

We isolated EPCs from the rat bone marrow and found that many cells showed a round, cobblestone-like morphology in the primary adherent cell culture. Furthermore, the majority of these primary cells showed uptake of DiI-AcLDL and FITC-UEA-I (Fig. 4A), suggesting that these cells were EPCs. In a flow cytometry analysis, we demonstrated that $67 \% \pm 3.2 \%$ of the cultured cells were $\mathrm{KDR}+, 49 \% \pm 3.6 \%$ were $\mathrm{CD} 34+$, and $35 \% \pm 1.8 \%$ were $\mathrm{KDR}+/ \mathrm{CD} 34+$. The flow cytometry analysis indicated that these $\mathrm{KDR}+/ \mathrm{CD} 34+$ cells were EPCs (Fig. 4B). 

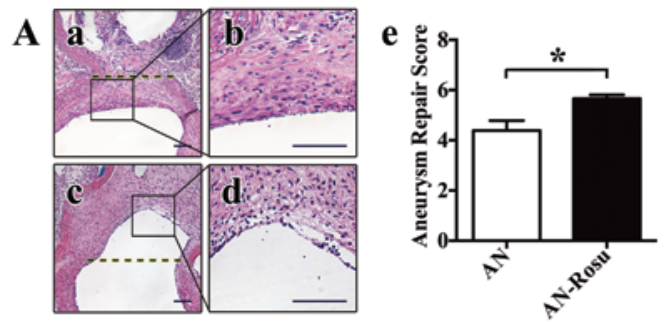

B

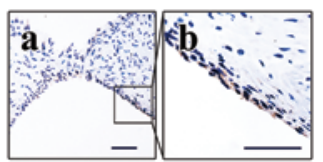

C

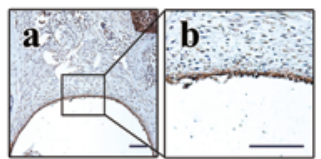

D

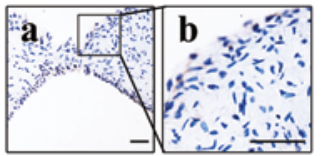

E

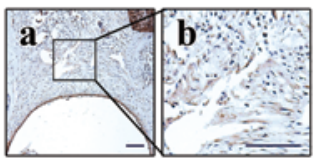

F

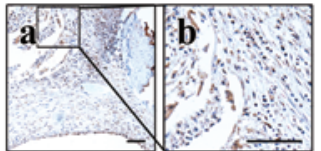

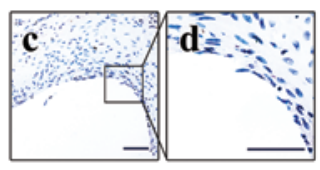
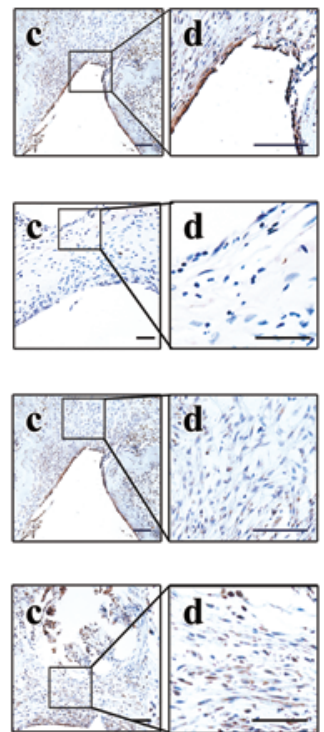

G

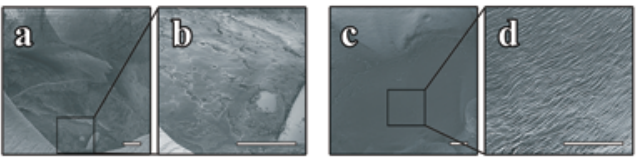

H
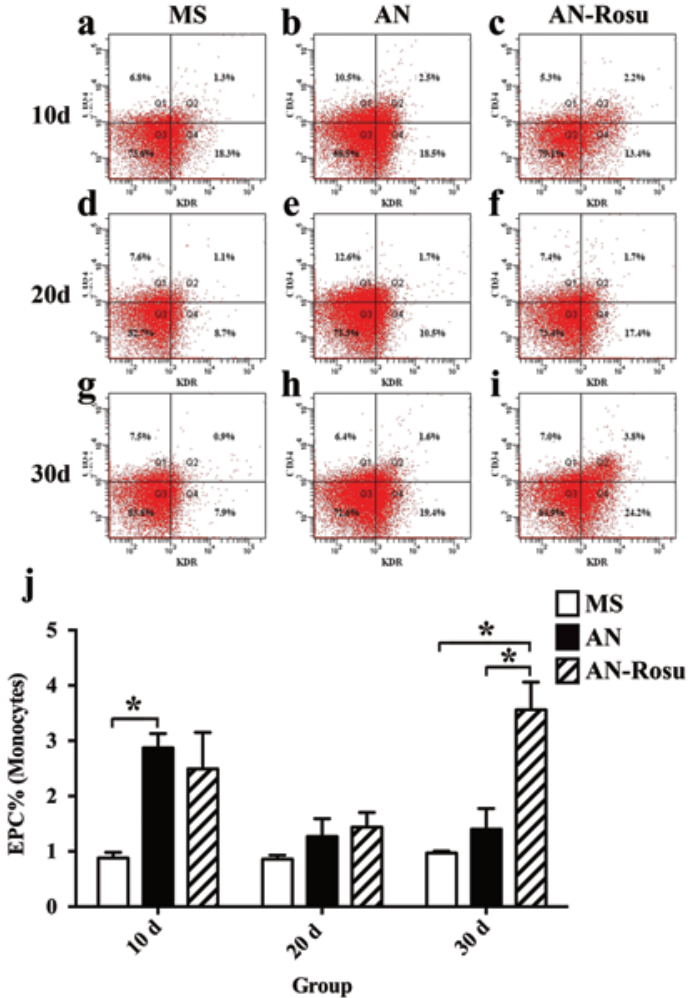

FIG. 3. Histological investigation and cell identification. A: Photomicrographs of $\mathrm{H}$ \& E-stained aneurysm neck sections obtained from a rat from the AN-Rosu group after 30 days of rosuvastatin treatment (a) and an untreated rat from the AN group at the same time point (c). The integration of the aneurysm neck can be clearly observed in the magnified images (b and d). Bar $=50 \mu \mathrm{m}$. The bar graph (e) shows the aneurysm repair score in the rosuvastatin-treated group and the controls. Data are the mean \pm SE calculated from assessment of 18 sections per group ( 3 sections randomly chosen from each of 6 animals). ${ }^{*} p<0.05$. B-F: The photomicrographs show KDR+ and vWF+ stains for the aneurysm neck in the rosuvastatin-treated AN-Rosu group (Ba and $\mathrm{Ca}$ ) and the untreated $\mathrm{AN}$ group $(\mathrm{Bc}$ and $\mathrm{Cc}$ ) following 30 days of rosuvastatin treatment. The number of $\mathrm{KDR}+$ cells increased $(\mathrm{Bb})$ and the vWF+ cells were more integrated into the aneurysm neck in the rosuvastatin-treated rats (magnified images) compared with findings in the untreated rats with aneurysms (Bd and $\mathrm{Cd}$ ). The photomicrographs show KDR+, vWF+, and $\alpha \mathrm{SMA}+$ stains for the lumen of the aneurysm sac in the AN-Rosu group following 30 days of rosuvastatin treatment ( $\mathrm{Da}$, Ea, and Fa) and AN group at the same time point (Dc, Ec, and Fc). Magnified images show that KDR, vWF, and $\alpha S M A$ expression were increased in the ANRosu group (Db, Eb, and Fb) compared with the AN group (Dd, Ed, and Fd). Bar $=50 \mu \mathrm{m}$. G: Scanning electron micrographs showing the endothelialization in the aneurysm neck. Consistent simple squamous epithelial cells at the bottom of the aneurysm neck were detected in the rosuvastatin-treated rats (a) but not in the untreated rats (c); a smaller area of each section is shown at higher magnification in $\mathbf{b}$ and $\mathbf{d}$. Bar $=100 \mu \mathrm{m}$. H: Circulating EPC levels on Days 10, 20, and 30 in the MS group (a, d, and $\mathbf{g}$ ), the AN group (b, e, and h), and the AN-Rosu group (c, $f$, and i) were determined via assessment of CD34/KDR double-positive cells. The bar graph (j) shows a quantitative analysis of CD34+/KDR+ cells in the control (MS) group and aneurysm with (ANRosu) and without rosuvastatin (AN) treatment groups. Data are the mean $\pm S E, n=5$ per group, ${ }^{*} p<0.05$, rosuvastatin group versus untreated group and control group.

\section{Effect of Rosuvastatin on EPC Viability and Functioning MTT Assays}

We showed that the viability of EPCs after 24 hours of rosuvastatin treatment increased in a dose-dependent manner that plateaued at $100 \mu \mathrm{mol} / \mathrm{L}$. We found that 100 $\mu \mathrm{mol} / \mathrm{L}$ of rosuvastatin treatment for 24 hours induced a marked increase in colony formation compared with the untreated cells. Based on this result, 24-hour treatment with $100 \mu \mathrm{mol} / \mathrm{L}$ rosuvastatin was selected for the following experiments (Table 3, Fig. 4C).

\section{Migration Assay}

Cell monolayers applied to wells with a scratch technique and treated for 24 hours with $100 \mu \mathrm{mol} / \mathrm{L}$ rosuvas- 
TABLE 2. EPC (monocyte) percentage*

\begin{tabular}{cccc}
\hline & \multicolumn{3}{c}{ Group } \\
\cline { 2 - 4 } Time & AN & AN-Rous & MS \\
\hline 10 days & $2.87 \pm 0.26 \dagger$ & $2.49 \pm 0.66$ & $0.88 \pm 0.10$ \\
\hline 20 days & $27 \pm 0.32$ & $1.44 \pm 0.26$ & $0.86 \pm 0.07$ \\
\hline 30 days & $1.40 \pm 0.37$ & $3.56 \pm 0.50 \dagger \ddagger$ & $0.97 \pm 0.03$ \\
\hline
\end{tabular}

* Values are percentages and represent the mean \pm SE $(n=5)$. Significant differences were assessed using 2-way ANOVA.

$\dagger$ Significantly different from MS group $(p<0.05)$

$\ddagger$ Significantly different from AN group $(p<0.05)$.

tatin showed greatly increased EPC migration $(\mathrm{p}<0.05$, Table 4, Fig. 4C).

\section{Tube Formation Assay}

Similar to the migration assay, we found that 24-hour treatment with $100 \mu \mathrm{mol} / \mathrm{L}$ rosuvastatin also increased EPC tube formation compared with the control $(p<0.05$, Table 4, Fig. 4C).

\section{Discussion}

In the present study, we successfully established a rat coiled aneurysm model via vasotransplantation and used MR angiography to confirm the feasibility of experimental aneurysm embolization. Rosuvastatin treatment was associated with a significant increase in the number of circulating EPCs in the rats with coil-treated aneurysms, and EPCs also participated in aneurysm neck endothelialization. The histological and SEM examinations demonstrated that rosuvastatin produced a robust effect on aneurysm neck closure and endothelialization. The experimental aneurysm repair score assessment system that we used in this study is the first such system to be reported, and the aneurysm repair score reflected the interaction between aneurysm embolization and coverage of endothelial cells, which is essential in preventing aneurysm recurrence. Although there was subjective bias during the assessment, the use of researchers who were blind to the conditions and assessment of multiple samples reduced this bias. The aneurysm repair score showed a trend of agreement with the assessment using $\mathrm{H} \& \mathrm{E}$ staining, and rosuvastatin also demonstrated robust promotion of EPC proliferation, migration, and tube formation in vitro.

The in vivo dosage of rosuvastatin that we chose was $20 \mathrm{mg} / \mathrm{kg} / \mathrm{day}$. We first tested concentrations of rosuvastatin in vitro and found that $100 \mu \mathrm{mol} / \mathrm{L}$ treatment reached the best therapeutic effect with the least unintended effects (Fig. 4). Considering the pharmacokinetics, disposition, and liver-specific distribution of rosuvastatin in vivo, we calculated the concentration, which ranged from 1 to 100 $\mu \mathrm{mol} / \mathrm{L}$. Additionally, the dosages of rosuvastatin previously used in the rat model were $2 \mathrm{mg} / \mathrm{kg} / \mathrm{day},{ }^{25} 5 \mathrm{mg} / \mathrm{kg} /$ day, ${ }^{19} 10 \mathrm{mg} / \mathrm{kg} / \mathrm{day}$, and $15 \mathrm{mg} / \mathrm{kg} / \mathrm{day} .{ }^{20}$ The dosage we used in vivo was within the range of previously reported studies. We used a single dosage in our study since the purpose of the study was to supply proof of the principle.

EPCs play an important role during vascular endothelium repair and remodeling, especially in vascular injury
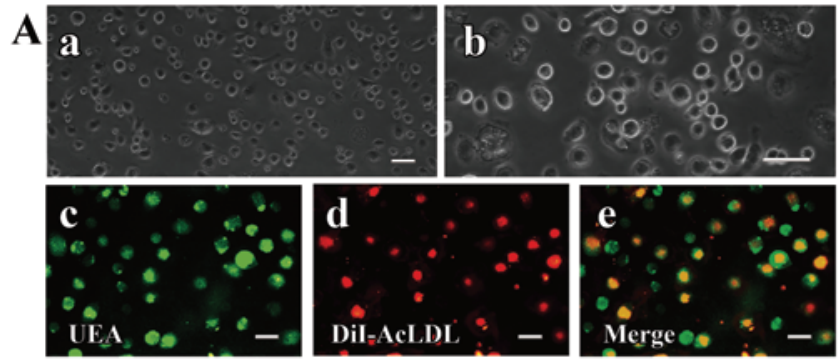

B
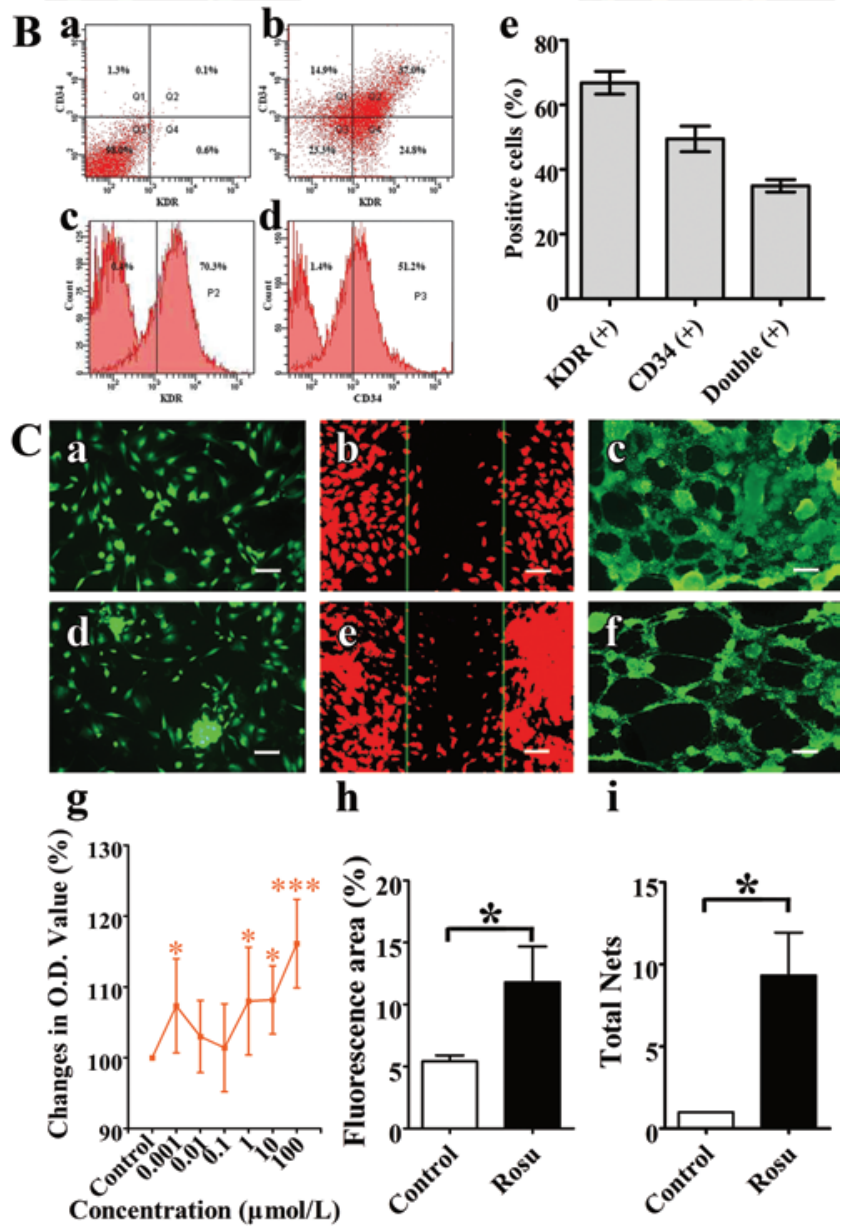

h
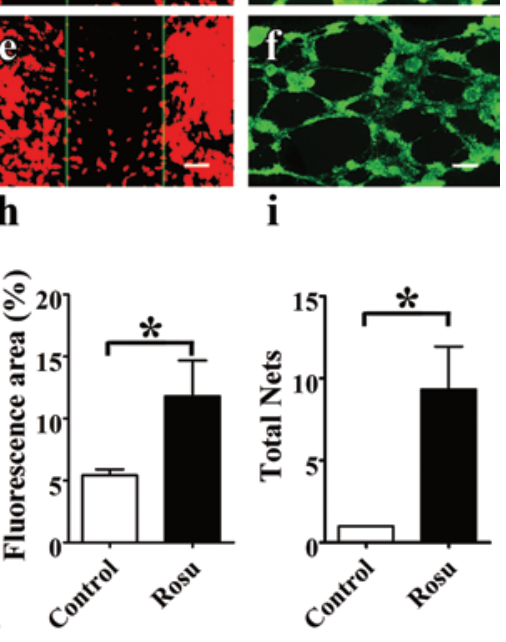

FIG. 4. Rosuvastatin promotion of EPC proliferation, migration, and tube formation. A: Photomicrographs showing the cultured EPCs that were used for EPC determination. Representative photomicrographs (a and b) show adherent primary cells after 10 days of culture (b magnified from a). $B a r=40 \mu \mathrm{m}$. Fluorescence photomicrographs (c-e) showing FITC-UEA-I (c) and Dil-AcLDL (d), and a merged image (e). B: CD34/ KDR double labeling (a and b) and labeling for KDR (c) and CD34 (d) alone following 10-day cell culture to identify EPCs. The bar graph shows a quantitative analysis of KDR+, CD34+, and KDR+/CD34+ cells. Data are the mean $\pm S E, n=6$ per group. C: Photomicrographs showing proliferation in calcein AM-labeled EPCs ( $a$ and d), migration in propidium iodide-labeled EPCs ( $b$ and $\mathbf{e}$ ), and tube formation in calcein AM-labeled EPCs (c and f) from the rosuvastatin-treated cultures (a, b, and c) and controls (d, e, and f). Bar $=40 \mu \mathrm{m}$. The bar graph shows EPC proliferation (g), migration (h), and tube formation (i) after $100-\mu \mathrm{mol} / \mathrm{L}$ rosuvastatin treatment. Data are the mean \pm SEM, $n=$ 5-6 per group; ${ }^{*} p<0.05,{ }^{* * *} p<0.001$, rosuvastatin group versus control group. 
TABLE 3. Optical density value elevation rate after 24-hour treatment with rosuvastatin

\begin{tabular}{cc}
\hline Concentration $(\mu \mathrm{mol} / \mathrm{L})$ & Change in OD Value $(\%)^{*}$ \\
\hline 0.001 & $107.3 \pm 2.3 \dagger$ \\
\hline 0.010 & $103.0 \pm 1.8$ \\
\hline 0.100 & $101.4 \pm 2.2$ \\
\hline 1.000 & $108.0 \pm 2.7 \dagger$ \\
\hline 10.000 & $108.2 \pm 1.7 \dagger$ \\
\hline 100.000 & $116.1 \pm 2.2 \dagger$ \\
\hline
\end{tabular}

* OD values are expressed as mean \pm SEM $(n=6)$. Significance was assessed by means of a 1-way ANOVA followed by Dunnett's t-test.

$\dagger$ Significantly different from control group $(p<0.05)$.

and coiled aneurysms, ${ }^{3,21}$ and EPCs have been found to promote aneurysm neck endothelialization after aneurysm coiling. ${ }^{12,27}$ In this study, we found that the number of circulating EPCs was significantly increased at 10 days after aneurysm coiling - an increase that could have been caused by bone marrow-derived EPC mobilization and homing after vascular injury. During this stage, increased levels of circulating EPCs were primarily ascribed to the vascular injury, but rosuvastatin appeared to produce an observable effect. On the 20th day, most of the circulating EPCs were hypothesized to have migrated to the injured aneurysm neck, targeting the impaired endothelium, thereby decreasing the number of circulating EPCs. Late stages of endothelium repair were present on the 30th day. In this stage, the number of local EPCs decreased, and the number of circulating EPCs recovered to a relatively high level. During this period, the rosuvastatin-treated rats showed significant increases in the number of circulating EPCs. Previous studies showed strong evidence indicating that statins mobilize EPCs from bone marrow to increase the amount of circulating EPCs. ${ }^{16,17}$

Tissue samples from the coiled aneurysms were assessed after 30 days of rosuvastatin therapy using an aneurysm repair score assessment system that we established. The rosuvastatin-treated rats showed better neurological outcomes than the untreated rats. Regarding the aneurysm repair score and histological examination, rosuvastatin treatment improved aneurysm neck integration and focal endothelialization. Staining for vWF further demonstrated the continuity of the endothelial cell layer in the aneurysm necks obtained from these rats. Similar to the aneurysm repair score, the rosuvastatin-treated rats had additional integrated endothelium compared with the untreated rats with aneurysms. KDR is a representative marker of EPCs, and we found that more KDR+ cells adhered to the impaired aneurysm neck in the rosuvastatin-treated rats, whereas few KDR+ cells were detected in the untreated rats with aneurysms. Our previous study demonstrated the effect of bone marrow-derived EPCs in aneurysm neck endothelialization..$^{30}$ In the present study, we further demonstrated that the numbers of circulating EPCs increased in the rosuvastatin-treated rats, and more KDR+ cells adhered to the endothelial layer in the aneurysm neck (Fig. 3 ), indicating that rosuvastatin could promote EPC attachment to the aneurysm neck. SEM examination demon-
TABLE 4. Results of migration and tube formation assays*

\begin{tabular}{lcc}
\hline \multicolumn{1}{c}{ Measure } & Rosuvastatin $(-)$ & Rosuvastatin $(+)$ \\
\hline $\begin{array}{l}\text { Fluorescent area of the migra- } \\
\text { tion assay }\end{array}$ & $5.4 \% \pm 0.5 \%$ & $11.8 \% \pm 2.9 \%{ }^{*} \dagger$ \\
\hline $\begin{array}{l}\text { Total no. of nets in the tube } \\
\text { formation assay }\end{array}$ & $1.0 \pm 0$ & $9.3 \pm 2.6^{*} \dagger$ \\
\hline * The values represent the mean \pm SE of 5 wells of EPCs for the migration \\
assay and mean \pm SE of 3 wells of EPCs for the tube formation assay. \\
$\dagger$ Significantly different from control group $(p<0.05,1$-way ANOVA).
\end{tabular}

strated that the bottom of the aneurysm neck had simple squamous epithelial cells in the rosuvastatin-treated rats (as opposed to spindle endothelial cells). These EPCs were thought to originate from circulating EPCs. Although we found an increase of circulating EPCs in the rosuvastatintreated animals, we could not show where the increased EPCs came from. As we know, 2 types of endothelial cells were found at the aneurysm neck. One type was squamous endothelial cells, which we found covering the bottom of the aneurysm neck. The other type was the slim and spindle endothelial cells, which surrounded the ring of the aneurysm neck. The latter may come from local neighbor endothelial cells since the cell morphological character was similar to that of the surrounding cells. Nevertheless, rosuvastatin promoted migration of endothelial cells and EPCs toward the aneurysm neck and contributed to its endothelialization.

There are several mechanisms that might have been responsible for the enhanced repair effect induced by the EPCs. First, rosuvastatin accelerated the EPC mobilization from bone marrow and increased the level of circulating EPCs after coiling treatment. Clinical research has shown that long-term statin therapy helps maintain high levels of circulating EPCs. ${ }^{7}$ Second, rosuvastatin directly promoted the viability of adherent EPCs and the function of endothelial cells. We found that rosuvastatin positively affected EPC proliferation, migration, and tube formation in rats in vivo, which indicated that the direct promotion of EPCs by rosuvastatin was another crucial component inducing aneurysm neck repair and remodeling. In addition to having a proliferative effect, statins have also been shown to exert positive effects on cell survival and differentiation by reducing apoptosis and suppressing apoptotic cytokines. ${ }^{15,29}$ Rosuvastatin facilitated local release of endothelium-related cytokines in aneurysm necks, which may promote endothelialization. In addition, the paracrine effect of EPCs may contribute to the acceleration of endothelialization. ${ }^{14}$ We intend to further explore the mechanism of action of rosuvastatin in the improvement of aneurysm neck endothelialization in future studies.

Previous studies included somewhat conflicting results. The pathological progress of coiled cerebral aneurysms has been shown to be affected by the interaction of many factors, including EPCs, smooth muscle cells, and inflammation. Although bone marrow-derived EPCs were shown not to be involved in the re-endothelialization of a mouse endothelial denudation model or murine arterial injury model,,${ }^{13}$ EPCs were thought to play a key role in the 
regulation of postinjury vascular endothelialization by secreting cytokines or microvesicles containing DNA, RNA, or microRNA. ${ }^{6,26}$ The beneficial effects of statins in clinical aneurysm treatment are also controversial. A singlecenter case-control study found no evidence of beneficial effect of statins with respect to intracranial aneurysm suppression. ${ }^{24}$ In contrast, the results of a Japanese multicenter case-control study suggested an inverse relationship between statin use and cerebral aneurysm rupture. ${ }^{28}$

\section{Conclusions}

The results of our study show that statins had a positive effect of aneurysm repair and indicate that further studies of the role of statins in aneurysm repair should be conducted.

\section{Acknowledgments}

This project was sponsored by the National Natural Science Foundation of China, project No. 30973105 (W.Z.); the Program for New Century Excellent Talents in University, project No. XYQ2011019 (W.Z.); and Shanghai Rising Star Program, project No. 13QH1400900 (W.Z.).

\section{References}

1. Aronson JP, Mitha AP, Hoh BL, Auluck PK, Pomerantseva I, Vacanti JP, et al: A novel tissue engineering approach using an endothelial progenitor cell-seeded biopolymer to treat intracranial saccular aneurysms. J Neurosurg 117:546-554, 2012

2. Asahara T, Murohara T, Sullivan A, Silver M, van der Zee R, $\mathrm{Li} \mathrm{T}$, et al: Isolation of putative progenitor endothelial cells for angiogenesis. Science 275:964-967, 1997

3. Balaji S, King A, Crombleholme TM, Keswani SG: The role of endothelial progenitor cells in postnatal vasculogenesis: implications for therapeutic neovascularization and wound healing. Adv Wound Care (New Rochelle) 2:283-295, 2013

4. Basile DP, Yoder MC: Circulating and tissue resident endothelial progenitor cells. J Cell Physiol 229:10-16, 2014

5. Dai D, Ding YH, Danielson MA, Kadirvel R, Lewis DA, Cloft HJ, et al: Modified histologic technique for processing metallic coil-bearing tissue. AJNR Am J Neuroradiol 26:1932-1936, 2005

6. Deregibus MC, Cantaluppi V, Calogero R, Lo Iacono M, Tetta C, Biancone L, et al: Endothelial progenitor cell derived microvesicles activate an angiogenic program in endothelial cells by a horizontal transfer of mRNA. Blood 110:24402448, 2007

7. Deschaseaux F, Selmani Z, Falcoz PE, Mersin N, Meneveau N, Penfornis A, et al: Two types of circulating endothelial progenitor cells in patients receiving long term therapy by HMG-CoA reductase inhibitors. Eur J Pharmacol 562:111118,2007

8. Di Stefano R, Felice F, Pini S, Mazzotta G, Bovenzi FM, Bertoli D, et al: Impact of depression on circulating endothelial progenitor cells in patients with acute coronary syndromes: a pilot study. J Cardiovasc Med (Hagerstown) 15:353-359, 2014

9. Erbs S, Beck EB, Linke A, Adams V, Gielen S, Kränkel N, et al: High-dose rosuvastatin in chronic heart failure promotes vasculogenesis, corrects endothelial function, and improves cardiac remodeling--results from a randomized, double-blind, and placebo-controlled study. Int J Cardiol 146:56-63, 2011

10. Frösen J, Marjamaa J, Myllarniemi M, Abo-Ramadan U, Tulamo R, Niemelä M, et al: Contribution of mural and bone marrow-derived neointimal cells to thrombus organization and wall remodeling in a microsurgical murine saccular aneurysm model. Neurosurgery 58:936-944, 2006

11. Ghani U, Shuaib A, Salam A, Nasir A, Shuaib U, Jeerakathil T, et al: Endothelial progenitor cells during cerebrovascular disease. Stroke 36:151-153, 2005

12. Groden C, Hagel C, Delling G, Zeumer H: Histological findings in ruptured aneurysms treated with GDCs: six examples at varying times after treatment. AJNR Am J Neuroradiol 24:579-584, 2003

13. Hagensen MK, Raarup MK, Mortensen MB, Thim T, Nyengaard JR, Falk E, et al: Circulating endothelial progenitor cells do not contribute to regeneration of endothelium after murine arterial injury. Cardiovasc Res 93:223-231, 2012

14. He T, Smith LA, Harrington S, Nath KA, Caplice NM, Katusic ZS: Transplantation of circulating endothelial progenitor cells restores endothelial function of denuded rabbit carotid arteries. Stroke 35:2378-2384, 2004

15. Henrich D, Seebach C, Wilhelm K, Marzi I: High dosage of simvastatin reduces TNF-alpha-induced apoptosis of endothelial progenitor cells but fails to prevent apoptosis induced by IL-1beta in vitro. J Surg Res 142:13-19, 2007

16. Hibbert B, Ma X, Pourdjabbar A, Simard T, Rayner K, Sun J, et al: Pre-procedural atorvastatin mobilizes endothelial progenitor cells: clues to the salutary effects of statins on healing of stented human arteries. PLoS One 6:e16413, 2011

17. Hibbert B, Simard T, Ramirez FD, Pourdjabbar A, Raizman JE, Maze R, et al: The effect of statins on circulating endothelial progenitor cells in humans: a systematic review. J Cardiovasc Pharmacol 62:491-496, 2013

18. Hill JM, Zalos G, Halcox JP, Schenke WH, Waclawiw MA, Quyyumi AA, et al: Circulating endothelial progenitor cells, vascular function, and cardiovascular risk. N Engl J Med 348:593-600, 2003

19. Hu X, Sun A, Xie X, Huang Z, Jia J, Yao R, et al: Rosuvastatin changes cytokine expressions in ischemic territory and preserves heart function after acute myocardial infarction in rats. J Cardiovasc Pharmacol Ther 18:162-176, 2013

20. Li W, Li Y, Zhu S, Ji Q, Shu Y, Zhang L, et al: Rosuvastatin attenuated the existing morphine tolerance in rats with L5 spinal nerve transection through inhibiting activation of astrocytes and phosphorylation of ERK42/44. Neurosci Lett 584:314-319, 2015

21. Liang C, Feng H, Deng BQ, Li ZF, Huang QH, Zhao W, et al: Decreased levels and function of circulating endothelial progenitor cells in unruptured intracranial saccular aneurysm patients. Neurol Sci 35:23-28, 2014

22. Long J, Wang S, Zhang Y, Liu X, Zhang H, Wang S: The therapeutic effect of vascular endothelial growth factor geneor heme oxygenase-1 gene-modified endothelial progenitor cells on neovascularization of rat hindlimb ischemia model. $\mathbf{J}$ Vasc Surg 58:756-65.e2, 2013

23. Marbacher S, Marjamaa J, Bradacova K, von Gunten M, Honkanen P, Abo-Ramadan U, et al: Loss of mural cells leads to wall degeneration, aneurysm growth, and eventual rupture in a rat aneurysm model. Stroke 45:248-254, 2014

24. Marbacher S, Schläppi JA, Fung C, Hüsler J, Beck J, Raabe A: Do statins reduce the risk of aneurysm development? A case-control study. J Neurosurg 116:638-642, 2012

25. Panchasara AK, Patel JC, Vadgama VK, Barvaliya MJ, Tripathi CB: Interaction between rosuvastatin and rocuronium in rat sciatic-gastrocnemius nerve-muscle preparation. J Anesth 28:727-732, 2014

26. Rehman J, Li J, Orschell CM, March KL: Peripheral blood "endothelial progenitor cells" are derived from monocyte/ macrophages and secrete angiogenic growth factors. Circulation 107:1164-1169, 2003

27. Tenjin H, Fushiki S, Nakahara Y, Masaki H, Matsuo T, Johnson CM, et al: Effect of Guglielmi detachable coils on 
experimental carotid artery aneurysms in primates. Stroke 26:2075-2080, 1995

28. Yoshimura Y, Murakami Y, Saitoh M, Yokoi T, Aoki T, Miura K, et al: Statin use and risk of cerebral aneurysm rupture: a hospital-based case-control study in Japan. J Stroke Cerebrovasc Dis 23:343-348, 2014

29. Zaitone SA, Abo-Gresha NM: Rosuvastatin promotes angiogenesis and reverses isoproterenol-induced acute myocardial infarction in rats: role of iNOS and VEGF. Eur J Pharmacol 691:134-142, 2012

30. Zhang S, An Q, Li Q, Huang J, Chen X, Chen X, et al: Therapeutic benefit of bone marrow-derived endothelial progenitor cell transplantation after experimental aneurysm embolization with coil in rats. PLoS One 9:e90069, 2014

\section{Disclosure}

The authors report no conflict of interest concerning the materi- als or methods used in this study or the findings specified in this paper.

\section{Author Contributions}

Conception and design: Zhu, Chen. Acquisition of data: Chen. Analysis and interpretation of data: Liu, Chen. Drafting the article: Liu. Critically revising the article: An, Huang, Yang. Reviewed submitted version of manuscript: Zhu, An, Huang, Yang. Approved the final version of the manuscript on behalf of all authors: Zhu. Statistical analysis: Liu, An. Administrative/ technical/material support: Liu. Study supervision: Yang.

\section{Correspondence}

Wei Zhu, Department of Neurosurgery, Huashan Hospital, Fudan University, Wulumuqi Zhong Rd., Shanghai 200040, China. email: drzhuwei@fudan.edu.cn. 